\title{
1 A Temporal Transcriptional Map of Human Natural Killer Cell
}

\section{Differentiation}

3

4 Aline Pfefferle ${ }^{1 \dagger}$, Herman Netskar ${ }^{2,3 \dagger}$, Eivind Heggernes Ask ${ }^{2,3}$, Susanne Lorenz ${ }^{4}$, Jodie P.

5 Goodridge ${ }^{2,3}$, Ebba Sohlberg ${ }^{1}$, Trevor Clancy ${ }^{2,3 \ddagger}$, Karl-Johan Malmberg $^{1,2,3^{*} \ddagger}$

6

$7{ }^{1}$ Center for Infectious Medicine, Department of Medicine Huddinge, Karolinska Institutet,

8 Stockholm, Sweden. ${ }^{2}$ Department of Cancer Immunology, Institute for Cancer Research, Oslo

9 University Hospital, Oslo, Norway. ${ }^{3}$ The KG Jebsen Center for Cancer Immunotherapy, Institute

10 of Clinical Medicine, University of Oslo, Oslo, Norway. ${ }^{4}$ Department of Tumor Biology,

11 Institute for Cancer Research, Oslo University Hospital, Oslo, Norway.

12

13 * Corresponding author.

14 †Shared first author.

$15 \$$ Shared last author. 


\section{Abstract}

17 Natural killer cell repertoires are functionally diversified as a result of differentiation, homeostatic

18 receptor-ligand interactions and adaptive responses to viral infections. However, the regulatory

19 gene-circuits that define the manifold cell states and drive NK cell differentiation have not been

20 clearly resolved. Here, we performed single-cell RNA sequencing of 26,506 cells derived from

21 sorted phenotypically-defined human NK cell subsets to delineate a tightly coordinated

22 differentiation process from a small population of $\mathrm{CD}_{5} 6^{\text {bright }}$ precursors to adaptive $\mathrm{NKG}^{2} \mathrm{C}^{+}$

23 CD56 $^{\text {dim }}$ NK cells. RNA velocity analysis identified a clear directionality in the transition from

$24 \mathrm{CD}^{\text {bright }}$ to $\mathrm{CD} 56^{\mathrm{dim}} \mathrm{NK}$ cells, which was dominated by genes involved in transcription and

25 translation as well as acquisition of NK cell effector function. Gene expression trends mapped to

26 pseudotime, defined by increasing entropy, identified three distinct transcriptional checkpoints,

27 reflecting important changes in regulatory gene-circuits. The CD56 ${ }^{\text {bright }} \mathrm{NK}$ cell population

28 dominated pseudotime with two distinct checkpoints separating precursors from intermediate states

29 that gradually took on transcriptional signatures similar to CD56 ${ }^{\mathrm{dim}} \mathrm{NK}$ cells. The final checkpoint

30 occurred during late terminal differentiation of $\mathrm{CD} 56^{\mathrm{dim}} \mathrm{NK}$ cells and was associated with unique

31 divergent gene-expression trends. Furthermore, we utilized this single-cell RNA sequencing

32 resource to decipher the regulation of genes involved in lysosomal biogenesis and found a

33 coordinated gradual increase in the $R A B 4$ and $B L O C 1 S$ gene families with differentiation into

34 CD56 $^{\mathrm{dim}} \mathrm{NK}$ cells. These results identify important gene programs driving functional

35 diversification and specialization during NK cell differentiation and hold potential to guide new

36 strategies for NK cell-based cancer immunotherapy. 


\section{Introduction}

38 Natural killer (NK) cells are innate lymphocytes that play a vital role in the immune response

39 through their ability to directly kill transformed and virus infected cells, and by orchestrating the

40 early phase of the adaptive immune response ${ }^{1}$. NK cells are commonly divided into two distinct

41 subsets based on their level of CD56 expression, eg. CD56 $6^{\text {bright }}$ and CD56 ${ }^{\mathrm{dim}} \mathrm{NK}$ cells, with distinct

42 functional properties. CD56 $6^{\text {bright }}$ NK cells, exhibiting an immunoregulatory role, are highly

43 responsive to cytokine stimulation, primarily located within secondary lymphoid organs and have

44 poor cytotoxic potential ${ }^{2-4}$. General consensus based on phenotypic profiling and functional and

45 transcriptional studies identifies this NK cell population as an immature precursor to CD56 ${ }^{\mathrm{dim}} \mathrm{NK}$

46 cells $^{5-9}$. CD56 $6^{\text {dim }}$ NK cells, making up $\sim 90 \%$ of all circulating NK cells, express CD16 and exhibit

47 higher cytotoxic potential coordinated through receptor-mediated input ${ }^{2,10}$. However, this is an

48 oversimplified view of the NK cell repertoire. Mass cytometry profiling of NK cell repertoires at

49 the single cell level has revealed an extensive phenotypic diversity comprising up to 100,000

50 unique subsets in healthy individuals ${ }^{11}$. Much of this diversity is based on combinatorial expression

51 of stochastically expressed germline encoded activating and inhibitory receptors that bind to MHC

52 and tune NK cell function in a process termed NK cell education ${ }^{10,12}$. Another layer of diversity

53 reflects the continuous differentiation through several well-defined intermediate phenotypes ${ }^{13}$ from

54 the naïve $\mathrm{CD} 56^{\text {bright }} \mathrm{NK}$ cells to the terminally differentiated, adaptive $\mathrm{NKG} 2 \mathrm{C}^{+} \mathrm{CD} 57^{+} \mathrm{CD} 56^{\text {dim }}$

55 NK cells, associated with past infection by cytomegalovirus $(\mathrm{CMV})^{14}$. Given the increasing interest

56 to harness the cytolytic potential of NK cells in cell therapy against cancer, it is of fundamental

57 importance to understand the molecular programs and regulatory gene circuits that drive NK cell

58 differentiation and underlie the functional diversification of the human NK cell repertoire.

Mouse studies identified important roles for T-bet and Eomes in the differentiation from

60 immature $\mathrm{CD} 27^{+} \mathrm{CD} 11 \mathrm{~b}^{-}$to mature $\mathrm{CD} 27^{-} \mathrm{CD} 11 \mathrm{~b}^{+} \mathrm{NK}$ cells, but did not delineate the exact 
61 intracellular signaling pathways mediating these effects ${ }^{15,16}$. In an attempt to characterize NK cell

62 heterogeneity within peripheral blood and organs using single-cell RNA sequencing (scRNA-seq),

63 Crinier et al., identified organ-specific signatures with four populations of human spleen NK cells

64 with gene signatures along a continuum confined by the traditional CD56 $6^{\text {bright }}$ and CD56 ${ }^{\text {dim }} \mathrm{NK}$ cell

65 subdivision ${ }^{17}$. Remarkably however, only two major transcriptional subsets were found in blood-

66 derived NK cells in both species. Through the use of bulk RNA and ChIP sequencing in human

67 NK cells, a TCF1-LEF-MYC axis was identified in CD56 ${ }^{\text {bright }}$ NK cells compared to CD56 ${ }^{\text {dim }}$ NK

68 cells where PRDM1 played a central role ${ }^{18}$. Furthermore, the CMV-driven adaptive NK cell

69 responses are associated with epigenetic modifications, ultimately reflected in CD8 T cell like

70 transcriptional profiles ${ }^{19-21}$. These gene regulatory programs underlying the CD56 $6^{\text {bright }}$ versus

71 CD56 $^{\mathrm{dim}} \mathrm{NK}$ cell phenotypic classification, provide a transcriptional basis for diverse functional

72 roles and localization of these subsets. However, it remains to be resolved how these major NK

73 cell subsets are related to other phenotypically defined stages of NK cell differentiation.

74 Phenotypically, NK cells are defined using a limited number of markers, but the true heterogeneity

75 of this population at the transcriptional level is unknown. Furthermore it remains to be examined

76 if NK cell differentiation at the transcriptional level is a linear process and if so, what transcriptional

77 checkpoints this may entail.

$78 \quad$ Here we used scRNA-seq and a combination of new bioinformatics tools to specifically

79 address the developmental relationship between distinct NK cell subsets and to map the gene

80 programs associated with transitions through discrete stages of differentiation. By sequencing

81 equal number of cells derived from five phenotypically well-defined human NK cell subsets, our

82 data unraveled a tightly coordinated differentiation process passing through a number of

83 transcriptional checkpoints, associated with unique gene-expression trends and changes in

84 functional modalities. By gaining a deeper understanding of the relationship between NK cell 

aCC-BY-NC 4.0 International license.

85 subsets and changes in genetic programs as cells transition through phases of NK cell education it

86 may be possible to develop new strategies to guide NK cell differentiation towards a desired

87 functional phenotype for cell therapy.

88 


\section{Results}

$90 \quad$ NK cell differentiation defined through single cell RNA-seq

91 To delineate the transcriptional landscape of human NK cell differentiation, we first performed

92 conventional bulk RNA sequencing of four sorted subsets representing distinct stages of NK cell

93 differentiation. PCA analysis identified a clear separation between CD56 ${ }^{\text {bright }}$, conventional and

94 adaptive CD56 ${ }^{\mathrm{dim}} \mathrm{NK}$ cells, whereas the two conventional CD56 ${ }^{\mathrm{dim}}$ subsets $\left(\mathrm{NKG}^{+} \mathrm{AIR}^{-}\right.$and

$95 \mathrm{NKG}^{-} \mathrm{A}^{-} \mathrm{KIR}^{+}$) were closer together in transcriptional space (Figure 1A). The four subsets were

96 ordered counter-clockwise in accordance with a model of NK cell differentiation laid out based on

97 proliferative responses to cytokines, which postulated that $\mathrm{CD}^{\text {bright }}$ differentiate into

$98 \mathrm{NKG}^{2} \mathrm{~A}^{+} \mathrm{KIR}^{-\mathrm{CD}} 56^{\mathrm{dim}}, \mathrm{NKG} 2 \mathrm{~A}^{-} \mathrm{KIR}^{+} \mathrm{CD} 56^{\mathrm{dim}}$ and finally to the most mature $\mathrm{NKG}^{\mathrm{A}} \mathrm{A}^{-}$

$99 \mathrm{KIR}^{+} \mathrm{NKG} 2 \mathrm{C}^{+} \mathrm{CD} 56^{\mathrm{dim}} \mathrm{NK}$ cells (the latter also termed adaptive). In order to address directionality

100 in this differentiation process and resolve the transition between these phenotypically defined cell

101 states, we performed single-cell RNA-sequencing (scRNA-seq) on bulk NK cells and five sorted

102 NK cell subsets, ranging from CD56 ${ }^{\text {bright }} \mathrm{NK}$ cells to distinct subsets of CD56 ${ }^{\text {dim }}$ NK cells,

103 including $\mathrm{CD}^{+} 7^{+} \mathrm{NKG} 2 \mathrm{C}^{+}$adaptive NK cells (Supplemental Figure 1A, 2, 3). In total, 26,506

104 cells were sequenced. Single cell transcriptional data from an equal number of sequenced cells

105 from each sample was pooled and merged in one single donor-specific t-SNE plot to examine the

106 relationship between phenotypically defined NK cell subsets across distinct stages of

107 differentiation (Supplemental Figure 1B). Because of the sorting strategy we were able to study

108 the rare CD56 ${ }^{\text {bright }} \mathrm{NK}$ cells and their relationship to CD56 ${ }^{\text {dim }} \mathrm{NK}$ cells in greater detail.

109 Importantly, despite the use of a limited set of markers to define the five sorted subsets, they

110 provided a complete representation of the total bulk NK cell signature as no bulk-specific cell

111 cluster was identified (Supplemental Figure 1B). 
113 bridge region (Figure 1B, Supplemental Figure 4A). Although the sorted NK cell subsets could

114 be ordered from left to right along the previously defined maturation scheme ${ }^{22}$, their transcriptomes

115 were highly overlapping with exception of the most naïve CD56 $6^{\text {bright }}$ and most mature adaptive

116 CD56 $^{\text {dim }}$ NK cell subsets. Unbiased clustering by PhenoGraph revealed two clusters in the

117 CD56 $6^{\text {bright }} \mathrm{NK}$ cell subset and two clusters in the conventional CD56 ${ }^{\mathrm{dim}} \mathrm{NK}$ cell subset (Figure 1B,

118 Supplemental Figure 4A). In the first donor, adaptive NK cells represented a fifth cluster as

119 defined by PhenoGraph that was not found in donor 2, lacking the adaptive NK cell subset.

120 Validation of the PhenoGraph algorithm using k-means clustering yielded similar results

121 (Supplemental Figure 1C). In agreement with previous data in bulk RNA-seq ${ }^{23}$, NK cells

122 expressing self and non-self KIR exhibited a high degree of transcriptional overlap and together

123 made up a significant portion of cluster 3 and 4 (Supplemental Figure 1D). Sorted

$124 \mathrm{NKG} 2 \mathrm{~A}^{+} \mathrm{CD} 56^{\mathrm{dim}} \mathrm{NK}$ cells exhibited high transcriptional variation and could be identified in all

125 PhenoGraph-defined clusters (Supplemental Figure 1D). Representation of the expression of

126 canonical differentiation markers, including NCAM (CD56), KLRC2 (NKG2C), SELL (CD62L),

$127 C D 7$ and $F C G R 3 A(\mathrm{CD} 16)$ across the t-SNE map corroborated the gradual transitioning from naïve

128 to more mature NK cells with progression from cluster 1 through 5 (Figure 1C).

129 To identify the gene signatures defining the five transcriptional clusters, we performed

130 differential gene expression analysis between clusters defining unique steps of NK cell

131 differentiation (Figure 1D-E). The most distal CD56 $6^{\text {bright }}$ cell population, confined within cluster

1321 were more transcriptionally diverse compared to cluster 2 cells and significantly increased genes

133 in cluster 1 included $I F N G, O A S 1, F G R, C D K 6, C C R 5$ and $S L C 37 A 1$. Visual representation of key

134 regulatory genes also revealed high expression of $I L 2 R B, I L 2 R G$ and $I L 15 R A$ as well as $K L R D 1$,

$135 R U N X 3$ and $I K Z F 2$ in cluster 1 (Figure 1F). The biggest transcriptional difference was observed 
136 between cluster 2 and 3, largely representing the CD56 $6^{\text {bright }}$ to $\mathrm{CD}^{\mathrm{dim}}$ transition, with $\mathrm{CD} 56^{\mathrm{dim}}$

137 NK cells being more transcriptionally diverse (Figure 1D-E). Genes with significantly increased

138 expression in cluster 2 included XCL1, SELL, CCR1, LEF1, IL7R, GZMK, LTB, CD27, CCR7,

$139 M Y C, C A P G, K I T, I L 23 A$ and $B A C H 2$. Conversely, cluster 3 was defined through higher expression

140 of CCL3, CCL4, CCL5, RORA, GZMA, GZMB, GZMH, GZMM, FCGR3A, TIGIT, NFKBIA,

$141 C X 3 C R 1, P R D M 1, Z E B 2, T F E B$ and $M I C B$. Cluster 3 and 4, containing a mixture of conventional,

142 phenotypically defined, CD56 ${ }^{\text {dim }}$ NK cell subsets, exhibited the fewest transcriptional differences

143 (Figure 1D-E) with CD38, LAIR2, GNAQ, RETSAT, CCDC41, BTRC and NARS2 being

144 upregulated in cluster 3 and only $A L K B H 2$ in cluster 4. Overall, cluster 3 appears to represent a

145 slightly more activated cell state within the conventional CD56 ${ }^{\text {dim }} \mathrm{NK}$ cell compartment with

146 higher expression of cytokine receptors, $C D 38$, SIGLEC7, KLRB1, and TBX21, rather than being

147 a unique differentiation stage (Figure 1F). The comparison between cluster 4 and 5 is

148 representative of the transition from conventional to adaptive NK cells and was characterized by a

149 general loss of gene expression (Figure 1D-E), in line with the previously reported epigenetic

150 reprogramming during terminal NK cell differentiation ${ }^{18,19}$.

151 Thus, analysis of scRNA-seq data from sorted NK cell subsets identified unique

152 transcriptional clusters, which only partially overlapped with phenotypic subsets. Notably, we

153 identified two transcriptionally-defined differentiation stages within the CD56 $6^{\text {bright }}$ population and

154 a unique cluster within the conventional CD56 $6^{\mathrm{dim}}$ population with a gene expression profile

155 suggestive of an activated cell state.

157 Continuous and coordinated transcriptional changes in pseudotime

158 Bulk RNA-seq of the two main NK cell populations has identified unique and evolutionary 159 conserved regulatory programs driven by TCF1-MYC (CD56 ${ }^{\text {bright }}$ ) and PRDM1-ZEB2-MAF 
$160\left(\mathrm{CD}^{\mathrm{dim}}\right)^{18}$. Plotting these regulatory genes on the transcriptional map, generated by merging

161 single-cell transcriptional signatures of distinct NK cell subsets, suggested that the TCF-MYC axis

162 is gradually replaced by the PRDM1-ZEB2-MAF-driven effector program (Figure 2A). Hence, we

163 hypothesised that these genes may be used to probe directionality and temporal relationships in the

164 differentiation process. Although sequencing of single cells only provides a snapshot in time, the

165 ratio of spliced to unspliced mRNA content within individual cells provides the necessary data to

166 calculate RNA velocity ${ }^{24}$. RNA velocity is a vector based on the time derivative of gene expression,

167 which can predict the future state of the cell (in the range of hours) in terms of gene expression.

168 Our dataset exhibited minimal differences in spliced and unspliced mRNA, with the exception of

169 within the CD56 $6^{\text {bright }}$ island (Figure 2B, Supplemental Figure 4B). Vector length, indicating the

170 speed at which the cells are changing gene expression, increased with proximity to the bridge

171 region linking the two transcriptional islands. Importantly, the directionality of the vector indicated

172 a transition from the CD56 $6^{\text {bright }}$ to the $\mathrm{CD} 56^{\mathrm{dim}}$ transcriptional island. Genes that contributed highly

173 to the RNA velocity vector, in terms of spliced versus unspliced mRNA, included genes associated

174 with transcription and translation $(M B N L 1, T N R C 6 B, P A R P 8, F O X P 1, N R 3 C 1)$, the actin

175 cytoskeleton ( $A R H G A P 15, U T R N, T X K)$, intracellular signaling (PRS3, TNIK), and NK cell

176 functionality ( $A O A H, C B L B, S K A P 1, C D 96, I L 12 R B 2, C L E C 2 D, F Y N, L Y S T)$ (Supplemental

177 Table 1).

178 Careful scrutiny of the subset origin of cells localized on both sides of the bridge suggests

179 a non-dramatic transition from CD56 $6^{\text {bright }}$ to CD56 ${ }^{\text {dim }}$ NK cells. A significant fraction of sorted

$180 \mathrm{NKG}^{2} \mathrm{~A}^{+} \mathrm{CD} 56^{\text {dim }} \mathrm{NK}$ cells were identified within the smaller predominantly CD56 ${ }^{\text {bright }}$ island

181 (Figure 1A, Supplemental Figure 4A). To further characterize the cells defining the bridge

182 region, we identified custom clusters (pre, post) consisting of the 100 most proximal cells on each

183 side of the bridge (Figure 2C). Despite $40 \%$ of the pre-cluster consisting of sorted CD56 ${ }^{\text {bright }}$ NK 
184 cells, the cluster also contained $50 \%$ sorted $\mathrm{NKG}_{2} \mathrm{~A}^{+} \mathrm{CD} 56^{\mathrm{dim}} \mathrm{NK}$ cells and even a small population

185 of $\mathrm{KIR}^{+} \mathrm{CD}^{\text {dim }} \mathrm{NK}$ cells (Figure 2D), suggesting that changes in these commonly used

186 phenotypic markers may be partly dissociated from underlying global transcriptional changes.

Thus, NK cell differentiation from CD56 ${ }^{\text {bright }}$ to $\mathrm{CD} 56^{\mathrm{dim}}$ is associated with coordinated and yet gradual changes in phenotypic surface markers that are tightly linked to reciprocal regulatory gene circuits controlled by NK cell-specific transcription factors.

192 To establish a transcriptional timeline of NK cell differentiation, we implemented pseudotime 193 (Palantir) analysis, providing an unbiased approach to model trajectories of differentiating cells ${ }^{25}$.

194 Palantir treats cell-fate as a probabilistic process and uses entropy to measure the changing nature 195 of cells along the differentiation trajectory. The starting cell (highest MYC expression) was chosen 196 based on the CD56 ${ }^{\text {bright }}$ regulome (Figure 2A, 3A, Supplemental Figure 4C) identified by Collins 197 et al ${ }^{18}$. Pseudotime analysis using BACH2 or LEF1 to define the starting cell yielded similar results.

198 The Palantir algorithm identified one terminal cell, located at the tip of cluster 5 furthest from the 199 bridge, belonging to the adaptive population (Figure 3A). In the conventional donor, the terminal 200 cell was identified within cluster 4, belonging to the mature population (Supplemental Figure 201 4C).

Plotting the transcriptional signatures of the sorted samples against pseudotime revealed

203 that $75 \%$ of pseudotime, reflecting gene diversity and decreasing entropy, was occurring within the

204 CD56 $6^{\text {bright }}$ NK cell differentiation stage (Figure 3B). Distribution within the CD56 ${ }^{\text {bright }}$ sorted cells

205 was centered around $65 \%$ of pseudotime, with the first $60 \%$ of pseudotime corresponding to the

206 smaller population of sorted cells grouping as cluster 1 cells (Figure 3B). This further corroborate

207 the notion that the immature CD56 $6^{\text {bright }}$ cells can be separated into two transcriptionally distinct 
subsets occupying distinct stages of the differentiation timeline (Figure 1A). Individual stages of

$209 \mathrm{CD}^{\mathrm{dim}}$ differentiation were largely assigned to the final $20 \%$ of pseudotime with outliers in the

$210 \mathrm{NKG} 2 \mathrm{~A}^{+} \mathrm{CD} 56^{\mathrm{dim}} \mathrm{NK}$ cells being part of the earlier phase in pseudotime (Figure 3B). NK cells

211 expressing self and non-self KIR occupied the same location within pseudotime whereas adaptive

212 NK cells were confined to the last 10\% of pseudotime (Figure 3B).

Having established a timeline for differentiation through the use of pseudotime,

214 generalized-additive models (GAMs) were fitted on cells ordered by pseudotime to identify

215 common gene expression. Three common global gene trends were identified, containing between

216713 to 1181 genes (Figure 3C). Distinct checkpoints (blue, green, red line) with low gene

217 expression diversity were identified in all gene trends at the same timepoints, indicative of

218 differentiation checkpoints (Figure 3C). In addition to low gene expression diversity, these

219 transcriptional checkpoints identified the timepoints where the gene expression changed

220 directionality. When mapped back to the t-SNE plot, the checkpoints were located at the tip of the

221 CD56 $6^{\text {bright }}$ island (blue), prior to the transition between cluster 1 and 2 (green) and at the transition

222 into the adaptive population (red) (Figure 3D). Hence major transcriptional changes are occurring

223 early after entering the NK cell lineage, during differentiation within the CD56 $6^{\text {bright }}$ NK population

224 and upon transitioning into the adaptive stage. Importantly, the checkpoint within the bright

225 population already occurs in the latter half of pseudotime, again highlighting that a dominating part

226 of transcriptional changes occur within the CD56 ${ }^{\text {bright }}$ population (Figure 3C).

228 programs associated with each global gene trend (Figure 3E). Trend 1 included genes whose

229 expression negatively correlated with pseudotime and which belonged to processes involved in

230 mitochondrial translational elongation, regulation of hematopoietic stem cell differentiation, TNF-

231 mediated signaling, and stimulatory C-type lectin receptor signaling. Genes included in trend 2 are 
232 initially stable but steadily increase from the bright checkpoint to the terminal checkpoint.

233 Transcriptional programs associated with this expression trend included intracellular signal

234 transduction, TGF $\beta$ receptor signaling, neutrophil degranulation, negative regulation of

235 transcription (RNA polymerase II) and actin cytoskeleton organization. The final trend, global

236 trend 3, contained genes whose expression decreased until the bright checkpoint and then remained

237 relatively stable until the terminal checkpoint. GO terms associated with these genes include

238 mRNA splicing (via spliceosome), apoptotic process, negative regulation of mitotic cell cycle

239 phase transition, mitochondrion organization and endomembrane system organization (Figure 3E).

241 Diversified gene-expression patterns during terminal NK cell differentiation

242 As the global pseudotime analysis was dominated by CD56 $6^{\text {bright }}$ NK cells we next zoomed in on

243 the later time-points ( $>80 \%$ of pseudotime), where higher standard deviation highlighted a poorer

244 fit of the identified global gene trends (Figure 3C). Hence, transcriptional programs within the

$245 \mathrm{CD}^{\mathrm{dim}}$ population were to a certain degree uncoupled from transcriptional programs defining NK

246 cell differentiation within the CD56 ${ }^{\text {bright }}$ stage. To dissect which transcriptional programs accounted

247 for this variation observed in the gene trends, we performed new clustering only taking pseudotime

$248>80 \%$ into account (Figure 4A). Three new CD56 ${ }^{\text {dim }}$ trends (dim trends) were identified,

249 consisting of two down-trending and one up-trending trend, in line with the general decrease in

250 gene expression observed in the transition from conventional CD56 ${ }^{\text {dim }}$ to adaptive cells ${ }^{19-21}$.

251 Dim trend 1, the largest trend in terms of gene number (2575 genes), contained genes that

252 steadily decreased within the later stages of pseudotime. Gene ontology terms associated with these

253 genes include mitochondrial translation elongation, apoptotic process, positive regulation of

254 telomere maintenance via telomerase, mitotic cell cycle process and regulation of hematopoietic 
255 stem cell differentiation (Figure 4B). Although initially decreasing, genes included in dim trend 2

256 were maintained at low expression from $90 \%$ of pseudotime onwards and associated with cellular

257 response to cytokine stimulus, positive regulation of leukocyte cell-cell adhesion, cell chemotaxis,

258 positive regulation of metabolic process and regulation of leukocyte differentiation. Lastly, dim

259 trend 3 contained genes which steadily increased in the final stages of pseudotime, representing a

260 clear minority. Gene ontology terms associated with these genes include leukocyte activation,

261 negative regulation of cellular process and regulation of intracellular signal transduction.

263 NK cells during education ${ }^{23}$. Self-KIR ${ }^{+}$NK cells show a non-transcriptional accumulation of

264 granzyme B stored in large dense-core secretory lysosomes, which are released upon target cell

265 recognition ${ }^{23}$. However, such dynamic changes to the lysosomal compartment with consequences

266 on the ability to store granzyme B and perforin, requires a continuous and transcriptionally-

267 regulated biogenesis of lysosomes and effector molecules as well as tight coordination of genes

268 involved in synapse formation and degranulation. Indeed, whereas the dominating gene expression

269 trends within the $\mathrm{CD} 56^{\mathrm{dim}}$ stages of differentiation ( $>80 \%$ of pseudotime) show a gradual decrease

270 in gene expression, genes involved in lysosomal biogenesis and effector function largely followed

271 dim trend 3, which increases with differentiation and thereby provide a template for further

272 functional tuning during NK cell education. We performed factor analysis on genes involved in

273 lysosomal biogenesis and projected these onto the temporal transcriptional map of human NK cell

274 differentiation (Figure 4C). Factor analysis revealed lowest expression in cluster 1, whereas the

$275 \mathrm{CD}^{\mathrm{dim}}$ clusters (cluster 3-5) exhibited the highest expression. Next, we zoomed in on individual

276 genes contained within the factor analysis which are important vesicular components and involved

277 in their regulation and biogenesis (BLOC complex) (Figure 4D). The IL-15 inducible genes $P R F 1$

278 (perforin), GZMA (granzyme A), and GZMB (granzyme B) were highly expressed in cluster 3, in 
279 line with higher $I L 15 R A$ and $I L 2 R G$ expression in this cluster (Figure 1E). GZMA, GZMB and

280 GZMH (granzyme H) were also highly expressed within cluster 5. SRGN (serglycin) and GZMK

281 (granzyme K) exhibited a reverse gene expression pattern, being highly expressed within cluster 1

282 and 2 . The Rab proteins $(R A B 4 A, R A B 4 B, R A B 27 A)$ play a role in vesicular and protein trafficking

283 as well as granule exocytosis, maturation and docking at the immune synapse. These genes

284 exhibited higher expression within the CD56 $6^{\mathrm{dim}}$ island, with some outliers within cluster 1 also

285 exhibiting higher expression. VAMP7 and STX7 are important for cytotoxic granule exocytosis in

286 NK cells and were highly expressed within cluster 1 and 3, again in line with increased IL-15

287 signaling in these cells. Lysosomal exocytosis is regulated through calcium signaling and at the

288 transcriptional level by $T F E B$, which is further regulated through phosphorylation ${ }^{26}$. TFEB

289 expression greatly increased after transitioning into the CD56 $6^{\mathrm{dim}}$ island, with highest expression

290 found in cluster 3. Lastly, the BLOC complex, consisting of BLOC1S1, BLOC1S2 and BLOC1S3,

291 was also higher expressed within the $\mathrm{CD} 56^{\mathrm{dim}}$ island. This complex is important for normal

292 biogenesis of lysosome-related organelles, such as granules and for their intracellular trafficking. 


\section{Discussion}

295 We report a compact description of the transcriptional diversification at the single cell level during

296 human NK cell differentiation. By enriching for less frequent, but phenotypically well-defined NK

297 cell subsets, we could elucidate key regulatory gene programs within both the CD56 $6^{\text {bright }}$ and the

298 CD56 $^{\mathrm{dim}}$ subset, as well as the developmental relationship of intermediate cell states. Pseudotime

299 analysis highlighted the dominant role CD56 $6^{\text {bright }} \mathrm{NK}$ cells play during NK cell differentiation, with

300 two out of three transcriptional checkpoints occurring within this small population of cells. In line

301 with previous reports on CMV-driven epigenetic reprogramming of terminally differentiated

302 adaptive NK cells ${ }^{19-21}$, the transition to this stage represented a major transcriptional checkpoint

303 during NK cell differentiation.

304 The view that NK cells, like T cells, undergo a continuous process of NK cell differentiation

305 is recent and mostly based on phenotypic and functional classification of discrete subsets ${ }^{5}$. Most

306 evidence suggest that the CD56 $6^{\text {bright }} \mathrm{NK}$ cell subset is the most naïve and gives rise to the more

307 mature CD56 ${ }^{\text {dim }} \mathrm{NK}$ cells which may then undergo further differentiation towards more terminal

308 stages, a process that is accelerated by CMV infection ${ }^{27}$. Numerous studies have been performed

309 in mice lacking NK specific transcription factors, using lineage tracing in macaques and in humans

310 with immunodeficiencies, but the transcriptional identities and relationships between the manifold

311 of putative intermediate cell states of NK cell differentiation remains elusive $\mathrm{e}^{5-9}$.

312 The CD56 $6^{\text {bright }} \mathrm{NK}$ cell subset has a unique functional phenotype and tissue localization.

313 Although infrequent in peripheral blood, they make up the large majority of NK cells within

314 secondary lymphoid organs ${ }^{4}$. In line with previous reports, t-SNE analysis of a comprehensive

315 scRNA-seq dataset from multiple sorted NK cell subsets identified two main transcriptional islands

316 of NK cells ${ }^{17}$. Whereas all CD56 $6^{\text {bright }} \mathrm{NK}$ cells were confined to the smaller island, a small but

317 definite collection of sorted CD56 ${ }^{\mathrm{dim}} \mathrm{NK}$ cells, primarily $\mathrm{NKG}^{2} \mathrm{~A}^{+} \mathrm{KIR}^{-} \mathrm{CD}^{-} 7^{-}$, grouped 
318 transcriptionally within the CD56 ${ }^{\text {bright }}$ population. While we cannot rule out the possibility of small

319 numbers of CD56 $6^{\text {bright }} \mathrm{NK}$ cells contaminating our sorted population, their low frequency within

320 the total NK cell population cannot account for the near $20 \%$ of CD56 ${ }^{\mathrm{dim}} \mathrm{NK}$ cells observed within

321 cluster 2. We have previously observed transcriptional reprogramming of educated NK cells to a

322 more immature transcriptional signature in response to IL-15 induced proliferation which also

323 correlated with NKG2A expression ${ }^{28}$. Hence, NKG2A ${ }^{+}$CD56 ${ }^{\text {dim }}$ NK cells exhibiting a CD56 bright

324 transcriptional signature could represent cells having undergone transcriptional reprogramming.

325 Alternatively, these cells could represent CD56 $6^{\text {bright }}$ NK cells that have downregulated CD56

326 expression at the surface level prior to further transcriptional changes occurring. CD56 is an

327 adhesion molecule and has been associated with formation of a developmental synapse and distinct

328 migratory behavior depending on the density of CD56 on the cell's surface ${ }^{29}$. Hence,

329 downregulation of CD56 surface expression could result in altered receptor input as a result of

330 synapse formation, ultimately leading to the acquisition of a CD56 ${ }^{\mathrm{dim}}$ transcriptional signature.

331 Although the bridge between transcriptional islands marked a clear decrease in NCAM1 (CD56)

332 expression, a gradual decreased was already observed prior to the bridge occurring within the late

333 CD56 $6^{\text {bright }} \mathrm{NK}$ cell population. FCERG3A (CD16) is normally expressed on CD56 ${ }^{\text {dim }}$ cells but can

334 also be used to define a population of functional intermediate CD56 $6^{\text {bright }}$ cells ${ }^{30}$. Although CD16

335 was not included in the sorting panel, FCERG3A is only significantly differentially expressed

336 between cluster 2 and 3, despite a moderate decrease from cluster 1 to 2 . Hence this functionally

337 intermediate $\mathrm{CD} 16^{+} \mathrm{CD} 56^{\text {bright }} \mathrm{NK}$ cell population may contribute but does not solely define the

338 two CD56 $6^{\text {bright }}$ clusters identified here.

339 RNA velocity, a recently described approach to predict future cell states ${ }^{24}$, confirmed a

340 transcriptional transition from CD56 $6^{\text {bright }}$ into CD56 $6^{\mathrm{dim}} \mathrm{NK}$ cells occurring via transition over the

341 bridge separating the two transcriptional islands. Among the top genes contributing to the RNA 
342 velocity vector were genes associated with NK cell receptor signaling and functionality (LYST,

$343 C B L B, C D 96, C L E C 2 D, S K A P 1, F Y N)$. LYST is a regulator of lysosomal trafficking, and mutations

344 in this gene leads to a lysosomal storage disorder, Chediak-Higashi syndrome, characterized by

345 defects in NK cell degranulation leading to the accumulation of large granules within these

346 patients ${ }^{31,32} \cdot C B L B$, has been linked to inhibitory NK cell signaling through its modulation of LAT,

347 a substrate of tyrosine phosphatase SHP- $1^{33}$. Hence, $\mathrm{Cbl}$ ubiquitin ligase encoded for by $C B L B$ is

348 important for mediating inhibitory receptor input which is essential for NK cell functionality.

349 Another inhibitory receptor on NK cells is CD96, which along with TIGIT and DNAM-1 can bind

350 PVR (CD155). CD96 is expressed at the protein level upon NK cell activation ${ }^{34}$ and competes with

351 DNAM-1 (CD226) for binding to their common ligand, with CD96 exhibiting an inhibitory

352 function leading to decreased cytokine release ${ }^{35}$. LLT1 (CLEC2D) is a C-type lectin that functions

353 as the ligand for CD161 (KLRB1). CD161 expression on NK cells has been linked to cytokine-

354 responsiveness and blocking of LLT1 enhanced cytotoxicity against breast cancer cells ${ }^{36,37}$. In T

355 cells, the adaptor protein SKAP-55 (SKAP1) links the T cell receptor to signaling via LFA-1 which

356 is also expressed on NK cells. Furthermore, $S K A P 1$ can also form homodimers and could play a

357 similar role in NK cells, where LFA-1 signaling has been linked to education ${ }^{38,39}$. Lastly, $F Y N$ is a

358 Src kinase involved in signaling through PI3K and ERK1/2, leading to increased cytotoxicity via

359 polarization of perforin in $\mathrm{NK}$ cells ${ }^{40}$. Transitioning across the bridge region was a gradual

360 transition phenotypically, with defining transcriptional changes, as identified by RNA velocity

361 occurring just prior to this region. In particular, these transcriptional changes occurred in genes

362 having major functional implications for NK cell cytotoxicity.

A surprising finding was the dominance of the CD56 $6^{\text {bright }}$ population in pseudotime and the

364 two transcriptionally unique clusters. The first $60 \%$ of pseudotime consisted of cluster 1 cells and

365 two of the three major transcriptional changes (checkpoints) occurred within this population and 
366 as it transitioned into cluster 2 cells. This highlights the transcriptional diversity within the

367 CD56 $6^{\text {bright }}$ population and identifies the group of cells at the beginning of pseudotime as

368 transcriptionally unique. This small population was characterized by high KLRB1 (CD161) KLRD1

369 (CD94), NCAM1 (CD56), CD7, IL15RA (CD215), IL2RB (CD122), IL2RG (CD132), RUNX3 and

$370 I K Z F 2$ (Ikaros) expression. IKZF2 has been shown to be important for NK cell precursors within

371 the liver ${ }^{41}$. Rather surprising, cells in cluster 1 at the beginning of pseudotime exhibited relatively

372 high levels of GZMB comparable to those in CD56 ${ }^{\text {dim }}$ NK cells. In mice, Granzyme B mRNA is

373 abundant in NK cells, but only translated upon cytokine stimulation ${ }^{42}$. In humans, CD56 ${ }^{\mathrm{dim}} \mathrm{NK}$

374 cells express high amounts of Granzyme B compared to CD56 $6^{\text {bright }}$ cells, but both can increase their

375 expression levels in response to cytokine stimulation ${ }^{43,44}$. High $G Z M B$ expression in this early

376 CD56 $6^{\text {bright }}$ precursor state could be due to high cytokine responsiveness, as these cells also

377 expressed high amounts of $I L 2 R B$ and $I L 2 R G$. NK cell development is also dependent on cytokine

378 priming, whereby $I L 2 R B$ and IL15RA knockout mice were deficient of NK cells ${ }^{45,46}$. To be able to

379 eliminate false-negatives (drop-outs) in our dataset, a result of technical limitations of scRNA-seq

380 combined with low RNA content in resting NK cells, we implemented MAGIC to help visualize

381 gene expression across the t-SNE map. Notably, MAGIC was not used for any other downstream

382 analysis, effectively avoiding the generation of false positives which can be introduced by such

383 imputation tools ${ }^{47}$.

384 The second transcriptional checkpoint was less defined in terms of standard deviation but

385 coincided with a change in gene expression within the trends analyzed. This correlated with

386 transitioning between cluster 1 and 2 within the $\mathrm{CD} 56^{\text {bright }} \mathrm{NK}$ cell population and was

387 characterized by a decrease in KLRB1 (CD161), KLRD1 (CD94), IL15RA (CD215), IL2RB

388 (CD122), IL2RG (CD132), RUNX3, IKZF2 (Ikaros), MYC and LEF1. The decrease in cytokine

389 receptors combined with $R U N X 3$ indicates reduced sensitivity to cytokine signaling, which is 
390 further reduced upon the CD56 $6^{\mathrm{dim}} \operatorname{transition}^{48}$. This is in line with CISH (CIS), a negative regulator

391 of IL-15 signaling, having the lowest expression in cluster $2^{49}$. Expression of effector molecule

392 genes, including PRF1 (perforin), GZMA (granzyme A), GZMB (granzyme B), GZMH (granzyme

$393 \mathrm{H})$ were also reduced in cluster 2. Overall, transitioning from cluster 1 to 2 was accompanied by a

394 decrease in transcriptional heterogeneity, resulting in a narrower transcriptional profile in these

395 later stages CD56 ${ }^{\text {bright }}$ cells.

396 Although the conventional CD56 ${ }^{\text {dim }}$ population exhibits a high degree of heterogeneity,

397 both phenotypically and functionally, it only consisted of two transcriptionally defined clusters,

398 clusters 3 and 4 . These two clusters had a similar distribution, mainly containing $\mathrm{KIR}^{+}$and a small

399 proportion of $\mathrm{NKG} 2 \mathrm{~A}^{+} \mathrm{CD} 56^{\mathrm{dim}} \mathrm{NK}$ cells. Both clusters exhibited higher expression of IKZF3

400 (Aiolos) and TBX21 (T-bet) compared to CD56 bright clusters, important transcription factors for

401 maturation of NK cells in the periphery ${ }^{15,50,51}$. When compared to cluster 4 , cluster 3 exhibited

402 increased gene expression which was indicative of an activated genotype, characterized by high

403 SIGLEC7, CD38, IL15RA (CD215), IL2RB (CD122) and PRF1 (Perforin) expression, rather than

404 a separate stage of differentiation ${ }^{52}$. NK cells express IL-15RA at detectable levels on the surface

405 after IL-15 stimulation ${ }^{53}$. Similarly, CD38 and Perforin are both upregulated upon IL-15

406 stimulation. Cluster 3 cells therefore appears to represent activated cells, which are responsive to

407 cytokine stimulation, in line with them occupying a slightly earlier pseudotime compared to cluster

408 4. In agreement with bulk RNA-seq in both mouse and human NK cells ${ }^{23,54}$, we observed no unique

409 transcriptional signatures between self KIR (educated) and non-self KIR (uneducated) NK cells

410 and both sorted populations occupied the same space in pseudotime ${ }^{23}$. Remodelling of the

411 lysosomal compartment has been shown to be important for the increased functionality observed

412 in educated NK cells ${ }^{23}$. Here, we observed an increase in lysosomal biogenesis in the later stages

413 of pseudotime, in line with increased functionality within the CD56 ${ }^{\text {dim }}$ NK cells. Thus, NK cell 
414 differentiation establishes a functional template through a tightly controlled and transcriptionally

415 regulated increase in the expression of genes involved in lysosomal biogenesis and exocytosis

416 alongside increased expression of effector molecules such as granzyme B and perforin. Acquisition

417 of self or non-self KIRs during later stages of differentiation sets the cells on different functional

418 trajectories during education, which involves a non-transcriptional remodelling of the lysosomal

419 compartment that ultimately changes the cytotoxic payload of the cell ${ }^{23}$.

$420 \quad$ Adaptive NK cells clustered independently and uniquely identified within the last $10 \%$ of

421 pseudotime. Transitioning into cluster 5 was accompanied by the third and final checkpoint,

422 highlighting the important transcriptional changes occurring at this stage of differentiation.

423 Compared to conventional CD56 ${ }^{\mathrm{dim}} \mathrm{NK}$ cells, the global transcriptome of adaptive NK cells was

424 highly reduced. This is in line with epigenetic silencing that has been described for this population

425 of terminally mature NK cells ${ }^{19-21}$. Gene expression of $S Y K, C D 38$ and $K L R B 1$ (CD161) was

426 reduced while $Z E B 2, K L F 2, P R D M 1$ (BLIMP-1), KLRC2 (NKG2C), GZMH (Granzyme H) were

427 highly expressed.

428 Here we have applied new bioinformatic tools to a unique single-cell RNA sequencing

429 dataset in order to identify a temporal transcriptional map of human NK cell differentiation.

430 Mapping gene expression trends to pseudotime allowed for the identification of distinct

431 transcriptional checkpoints highlighting important transcriptional changes during NK cell

432 differentiation. Two previously undescribed transcriptional populations within the CD56 $6^{\text {bright }}$

433 subset were identified and dominated the differentiation timeline. This dataset provides a valuable

434 tool to identify important gene programs that drive functional diversification and specialisation

435 during NK cell differentiation. Such knowledge hold potential to guide the development of new

436 strategies for NK cell-based cancer immunotherapy. 


\section{Materials \& Methods}

438 Cell processing

439 Peripheral mononuclear cells (PBMC) were isolated using density gradient centrifugation from

440 anonymized healthy blood donors (Oslo University Hospital) with informed consent as approved

441 by the regional ethics committee in Norway (scRNA-seq) and Sweden (bulk RNA-seq)

442 (2015/2095, 2016/1415-32, 2018/2485). Donor-derived PBMCs were screened for KIR education

443 and adaptive status using flow cytometry. NK cells were purified using an AutoMACS (DepleteS

444 program, Miltenyi Biotec) and prior to overnight resting in complete RPMI (10\% Fetal calf serum,

$4452 \mathrm{mM}$ L-glutamine) at $37^{\circ} \mathrm{C} / 5 \% \mathrm{CO}_{2}$.

$447 \quad$ Flow cytometry screening

448 PBMC were stained for surface antigens and viability in a 96 V-bottom plate, followed by

449 fixation/permeabilization and intracellular staining at $4^{\circ} \mathrm{C}$. The following antibodies were used in

450 the screening panel: CD3-V500 (UCHT1), CD14-V500 (M $\varphi$ P9), CD19-V500 (HIB19), Granzyme

451 B-AF700 (GB11) from Beckton Dickinson; CD57-FITC (HNK-1), CD38-BV650 (HB-7),

452 KIR3DL1-BV421 (DX9) from BioLegend; KIR2DL1-APC-Cy7 (REA284), CD158a,h-PE-Cy7

453 (11PB6), from Miltenyi Biotec; CD158b1/b2,j-PE-Cy5.5 (GL183), NKG2A-APC (Z199), CD56-

454 ECD (N901) from Beckman Coulter. LIVE/DEAD Fixable Aqua Dead Stain kit for $405 \mathrm{nM}$

455 excitation (Life Technologies) was used to determine viability. Samples were acquired on an LSR-

456 Fortessa equipped with a blue, red and violet laser and analyzed in FlowJo version 9 (TreeStar,

457 Inc.). 
FACS sorting

460 Cells were harvested and surface stained with the following antibodies: CD57-FITC (HNK-1) from

461 BioLegend; KIR3DL1S1-APC (Z27.3.7), CD56-ECD (N901), CD158b1/b2,j-PE-Cy5.5 (GL183),

462 from Beckman Coulter; KIR2DL1-APC-Cy7 (REA284), NKG2C-PE (REA205), NKG2A-PE

463 Vio770 (REA110) from Miltenyi Biotec. 12,000 cells were directly sorted into Eppendorf tubes at

$464 \quad 4^{\circ} \mathrm{C}$ for each sample (Supplemental Figure 1A) using a FACSAriaII (Beckton Dickinson).

465

466 Bulk RNA sequencing

467 Four populations (CD56 bright, NKG2A-KIR-CD56 ${ }^{\mathrm{dim}}, \mathrm{NKG}^{-} \mathrm{A}^{-} \mathrm{KIR}^{+} \mathrm{CD} 56^{\mathrm{dim}}$, and $\mathrm{NKG}^{-} \mathrm{A}^{-}$

$\left.468 \mathrm{KIR}^{+} \mathrm{NKG} 2 \mathrm{C}^{+} \mathrm{CD} 56^{\mathrm{dim}}\right)$ were sorted from six individual healthy blood donors. Sequencing was

469 performed using single-cell tagged reverse transcription (STRT) ${ }^{55}$. Principle component analysis

470 (PCA) was used to generate a biplot of the four sequenced subsets.

$472 \quad$ Single-cell RNA sequencing

473 Following sorting, cells were kept on ice during the washing (PBS $+0.05 \%$ BSA) and counting

474 step. 10,000 cells were resuspended in $35 \mu \mathrm{L}(\mathrm{PBS}+0.05 \% \mathrm{BSA})$ and immediately processed at

475 the Genomics Core Facility (Oslo University Hospital) using the Chromium Single Cell 3' Library

$476 \&$ Gel Bead Kit v2 (Chromium Controller System, 10X Genomics). The recommended 10x

477 Genomics protocol was used to generate the sequencing libraries, which was performed on a

478 NextSeq500 (Illumina) with 5 \% PhiX as spike-inn. Sequencing raw data were converted into

479 fastq files by running the Illumina`s bcl2fastq v2. 
482 Data cleaning steps were first carried out whereby cells not expressing a minimum of 1000

483 molecules and genes expressed by less than 10 cells were filtered out. The data was normalized

484 using log transformation based on the total expression of the gene in the sample, the default 485 normalization method implemented in the Palantir library. Feature selection was carried out to

486 select high cell-to-cell variance, implementing a cutoff of $\log 2$-fold change $>2$, whereby only

487 significantly differentially expressed genes were selected.

Dimensionality reduction of scRNA-seq data

490 A number of dimensionality reduction methods were implemented, including principle component

491 analysis (PCA) for initial noise reduction and non-linear diffusion maps to estimate a lower

492 dimensional manifold that could be implemented for further downstream analysis. For 493 visualization purposes, t-SNE and UMAP were utilized ${ }^{56,57}$.

496 Markov affinity-based graph imputation of cells (MAGIC) was utilized to de-noise the data in order

497 to optimize the gene expression analysis for visualization on the t-SNE maps ${ }^{58}$. The imputed data

498 matrix was not used for further downstream analysis and was not used for computation of the

499 differentially expressed genes (DEG).

500

501 Differentiation trajectories and gene trend analysis of scRNA-seq data 
502 Palantir was used to carry out the trajectory analysis and pseudotime calculations ${ }^{25}$. The starting

503 cells was identified as having the highest MYC expression, for which the imputed data matrix was

504 used to eliminate selection of outlier cells. The Palantir algorithm calculates the probability of each

505 individual cell to end up in each of the inferred terminal states, whereby only one terminal state

506 was identified in both donors. Generalized-additive models (GAMs) fitted on cells ordered by

507 pseudotime were used to calculate gene trends, where the contribution of cells was weighted by

508 their probability to end up in the given terminal state as calculated by Palantir. The gene trends

509 indicate how gene expression levels develop over the differentiation timeline. Local gene trends

510 were calculated by zooming in on a particular range of pseudotime $(>0.8)$.

512 Clustering, differential gene expression and gene set enrichment analysis scRNA-seq data

513 The gene trends were clustered using the PhenoGraph algorithm and confirmed using k-means

514 clustering, whereby the number of clusters identified by PhenoGraph was utilized as the k input

515 parameter $^{59}$. For differential gene expression (DEG) analysis, the SCDE package implementing the

516 Bayesian approach, was utilized ${ }^{60}$. SCDE is optimized to deal with the single cell specific challenge

517 of dropouts. A log-fold change of $>2$ and adjusted p-value $>0.05$ were deemed significant. Gene

518 over-expression analysis (Gene Ontology, PANTHER) was used downstream of the gene trend

519 clustering and DEG analysis to identify significant differences in biological pathways.

521 RNA velocity of scRNA-seq data

522 RNA velocity was run directly on the output created by Cell Ranger, containing the count matrix 523 and the abundance of spliced and unspliced versions of each transcript ${ }^{24}$. Using the velocyto Python 
524 library, the velocity vectors and locally average vector fields were calculated, which were the

525 projected onto the same t-SNE or UMAP embedding that was used for visualizing other analysis.

527 Factor analysis of scRNA-seq data

528 Factor analysis was utilized to obtain a single metric for a set of genes. The gene lists for specific

529 biological functions were obtained from Gene Ontology terms and the f-scLVM method in the

530 Python package slalom was then used for the factor analysis ${ }^{61}$.

532 Data sharing statement

533 All sequencing data (bulk and sc) will be deposited at NCBI GEO depository and will be

534 accessible with an accession number GEO: X or using the link

535 https://www.ncbi.nlm.nih.gov/geo/query/X.

537 Authorship and conflict-of-interest

538 A.P. performed the single-cell RNA sequencing experiments and the bulk RNA sequencing

539 experiments. S. L. performed the RNA sequencing and library preparation. H.N. and T.C.

540 performed the bioinformatic analysis. E.H.A. analyzed data. J.P.G and E.S. provided scientific

541 input. H.N. and T.C. contributed to the writing of the paper. H.N., T.C, A.P and K-J.M. designed

542 research. A.P. and K-J.M. wrote the manuscript. K-J.M. is a scientific advisor and consultant at

$543 \quad$ Fate Therapeutics. 
bioRxiv preprint doi: https://doi.org/10.1101/630657; this version posted May 7, 2019. The copyright holder for this preprint (which was not certified by peer review) is the author/funder, who has granted bioRxiv a license to display the preprint in perpetuity. It is made available under aCC-BY-NC 4.0 International license.

\section{Code availability}

546 Custom code utilized for analysis is available on the GitHub repository,

547 https://github.com/hernet/SingleFlow. 


\section{$548 \quad$ References}

549

550 1. Moretta A, Bottino C, Mingari MC, Biassoni R, Moretta L. What is a natural killer cell?

$551 \quad$ Nat Immunol. 2002;3(1):6-8.

552 2. Cooper MA, Fehniger TA, Caligiuri MA. The biology of human natural killer-cell subsets.

$553 \quad$ Trends Immunol. 2001;22:633-640.

554 3. Poli A, Michel T, Thérésine M, Andrès E, Hentges F, Zimmer J. CD56bright natural killer

555 (NK) cells: an important NK cell subset. Immunology. 2009;126:458-465.

556 4. Michel T, Poli A, Cuapio A, et al. Human CD56 bright NK Cells: An Update. J Immunol. $557 \quad 2016 ; 196: 2923-2931$.

558 5. Freud AG, Yokohama A, Becknell B, et al. Evidence for discrete stages of human natural 559 killer cell differentiation in vivo. J Exp Med. 2006;203(4):1033-1043.

560 6. Mace EM, Hsu AP, Monaco-Shawver L, et al. Mutations in GATA2 cause human NK cell

561 deficiency with specific loss of the CD56bright subset. Blood. 2013;121(14):2669-2678.

562 7. Maciejewski-Duval A, Meuris F, Bignon A, et al. Altered chemotactic response to

563 CXCL12 in patients carrying GATA2 mutations. J Leukoc Biol. 2016;99:1065-1076.

564 8. Gineau L, Cognet C, Kara N, et al. Partial MCM4 deficiency in patients with growth

565 retardation, adrenal insufficiency, and natural killer cell deficiency. J Clin Invest.

$566 \quad 2012 ; 122(3): 821-832$.

567 9. Wu C, Li B, Lu R, et al. Clonal tracking of rhesus macaque hematopoiesis highlights a

568 distinct lineage origin for natural killer cells. Cell Stem Cell. 2014;14(4):486-499.

569 10. Goodridge JP, Önfelt B, Malmberg K-J. Newtonian cell interactions shape natural killer $570 \quad$ cell education. Immunol Rev. 2015;267:197-213.

571 11. Horowitz A, Strauss-Albee DM, Leipold M, et al. Genetic and environmental determinants 
of human NK cell diversity revealed by mass cytometry. Sci Transl Med. 2013;5(208):1-

573 12.

574 12. Elliott JM, Wahle JA, Yokoyama WM. MHC class I-deficient natural killer cells acquire a $575 \quad$ licensed phenotype after transfer into an MHC class I-sufficient environment. J Exp Med. 2010;207(10):2073-2079.

577 13. Björkström NK, Riese P, Heuts F, et al. Expression patterns of NKG2A, KIR, and CD57 define a process of CD56dim NK-cell differentiation uncoupled from NK-cell education.

580 14. Sun JC, Beilke JN, Lanier LL. Adaptive immune features of natural killer cells. Nature. $2009 ; 457: 557-561$.

582 15. Gordon SM, Chaix J, Rupp LJ, et al. The Transcription Factors T-bet and Eomes Control Key Checkpoints of Natural Killer Cell Maturation. Immunity. 2012;36:55-67.

584 16. Ranson T, Vosshenrich CAJ, Corcuff E, et al. IL-15 availability conditions homeostasis of 585 peripheral natural killer T cells. Proc Natl Acad Sci U S A. 2003;100(5):2663-2668.

586 17. Crinier A, Milpied P, Escalière B, et al. High-Dimensional Single-Cell Analysis Identifies 587 Organ-Specific Signatures and Conserved NK Cell Subsets in Humans and Mice. Immunity. 2018;49:1-16.

589 18. Collins PL, Cella M, Porter SI, et al. Gene Regulatory Programs Conferring Phenotypic Identities to Human NK Cells. Cell. 2019;176(1-2):348-360.

591 19. Schlums H, Cichocki F, Tesi B, et al. Cytomegalovirus Infection Drives Adaptive 592 Epigenetic Diversification of NK Cells with Altered Signaling and Effector Function. Immunity. 2015;42(3):443-456.

594 20. Lau CM, Adams NM, Geary CD, et al. Epigenetic control of innate and adaptive immune 595 memory. Nat Immunol. 2018;19:963-972. 
596 21. Wiencke JK, Butler R, Hsuang G, et al. The DNA methylation profile of activated human

597 natural killer cells. Epigenetics. 2016;11(5):363-380.

598 22. Björkström NK, Riese P, Heuts F, et al. Expression patterns of NKG2A, KIR, and CD57

599 define a process of CD56dim NK cell differentiation uncoupled from NK-cell education.

$600 \quad$ Blood. 2010;116(19):3853-3864.

601 23. Goodridge JP, Jacobs B, Saetersmoen ML, et al. Remodeling of secretory lysosomes

602 during education tunes functional potential in NK cells. Nat Commun. 2019;10:1-15.

603 24. La Manno G, Soldatov R, Zeisel A, et al. RNA velocity of single cells. Nature.

$604 \quad 2018 ; 560(7719): 494-498$.

605 25. Setty M, Kiseliovas V, Levine J, Gayoso A, Mazutis L, Pe'er D. Palantir characterizes cell 606 fate continuities in human hematopoiesis. BioRxiv. 2018.

607 26. Sterea AM, Almasi S, El Hiani Y. The hidden potential of lysosomal ion channels: A new 608 era of oncogenes. Cell Calcium. 2018;72(January):91-103.

609 27. Liu LL, Pfefferle A, Yi Sheng VO, et al. Harnessing adaptive natural killer cells in cancer 610 immunotherapy. Mol Oncol. 2015;9(10):1904-1917.

611 28. Pfefferle A, Jacobs B, Ask EH, et al. Intra-lineage Plasticity and Functional 612 Reprogramming Maintain Natural Killer Cell Repertoire Diversity. bioRxiv. 2019:1-45.

613 29. Mace EM, Gunesch JT, Dixon A, Orange JS. Human NK cell development requires 614 CD56-mediated motility and formation of the developmental synapse. Nat Commun. $615 \quad 2016 ; 7(12171): 1-13$

616 30. Béziat V, Duffy D, Nguyen Quoc S, et al. CD56brightCD16+ NK Cells: A Functional 617 Intermediate Stage of NK Cell Differentiation. J Immunol. 2011;186:6753-6761.

618 31. Chiang SCC, Wood SM, Tesi B, et al. Differences in granule morphology yet equally 619 impaired exocytosis among cytotoxic T cells and NK cells from Chediak-Higashi 
syndrome patients. Front Immunol. 2017;8:1-15.

621 32. Jessen B, Maul-Pavicic A, Ufheil H, et al. Subtle differences in CTL cytotoxicity

622 determine susceptibility to hemophagocytic lymphohistiocytosis in mice and humans with

623 Chediak-Higashi syndrome. Blood. 2011;118(17):4620-4629.

624 33. Matalon O, Barda-Saad M. Cbl ubiquitin ligases mediate the inhibition of natural killer

625 cell activity. Commun Integr Biol. 2016;9(6):1-4.

626 34. Holmes VM, De Motes CM, Richards PT, et al. Interaction between nectin-1 and the

627 human natural killer cell receptor CD96. PLoS One. 2019;14(2):1-19.

628 35. Chan CJ, Martinet L, Gilfillan S, et al. The receptors CD96 and CD226 oppose each other 629 in the regulation of natural killer cell functions. Nat Immunol. 2014;15(5):431-438.

630 36. Kurioka A, Cosgrove C, Simoni Y, et al. CD161 defines a functionally distinct subset of 631 pro-inflammatory natural killer cells. Front Immunol. 2018;9(486):1-14.

632 37. Marrufo AM, Mathew SO, Chaudhary P, Malaer JD, Vishwanatha JK, Mathew PA.

633 Blocking LLT1 (CLEC2D, OCIL)-NKRP1A (CD161) interaction enhances natural killer 634 cell-mediated lysis of triple-negative breast cancer cells. Am J Cancer Res.

$635 \quad 2018 ; 8(6): 1050-1063$.

636 38. Raab M, Strebhardt K, Rudd CE. Immune adaptor protein SKAP1 (SKAP-55) forms 637 homodimers as mediated by the N-terminal region. BMC Res Notes. 2018;11(869):1-5.

638 39. Enqvist M, Ask EH, Forslund E, et al. Coordinated Expression of DNAM-1 and LFA-1 in Educated NK Cells. J Immunol. 2015;194:4518-4527.

640 40. Oykhman P, Timm-McCann M, Xiang RF, et al. Requirement and Redundancy of the Src 641 Family Kinases Fyn and Lyn in Perforin-Dependent Killing of Cryptococcus neoformans 642 by NK Cells. Infect Immun. 2013;81(10):3912-3922.

643 41. Boggs SS, Trevisan M, Patrene K, Geogopoulos K. Lack of natural killer cell precursors in 
fetal liver of Ikaros knockout mutant mice. Nat Immun. 1998;16:137-145.

645 42. Fehniger TA, Cai SF, Cao X, et al. Article Acquisition of Murine NK Cell Cytotoxicity

646 Requires the Translation of a Pre-existing Pool of Granzyme B and Perforin mRNAs.

$647 \quad$ Immunity. 2007;26:298-811.

648 43. Salcedo T, Azzoni L, Wolf S, Perussia B. Modulation of perforin and granzyme messenger

649 RNA expression in human natural killer cells. J Immunol. 1993;151:2511-2520.

650 44. Huang C, Bi E, Hu Y, et al. A Novel NF- kB Binding Site Controls Human Granzyme B

651

Gene Transcription. J Immunol. 2006;176:4173-4181.

652 45. Liu CC, Perussia B, Young JDE. The emerging role of IL-15 in NK-cell development.

653 Trends Immunol Today. 2000;21(3):113-116.

654 46. Suzuki H, Duncan GS, Takimoto H, Mak TW. Abnormal Development of Intestinal

655

Intraepithelial Lymphocytes and Peripheral Natural Killer Cells in Mice Lacking the IL-2

656

Receptor $\beta$ Chain. J Exp Med. 1997;185(3):499-506.

657 47. Andrews TS, Hemberg M. False signals induced by single-cell imputation.

$658 \quad$ F1000Research. 2018;7(1740):1-29.

659 48. Levanon D, Negreanu V, Lotem J, et al. Transcription Factor Runx3 Regulates

660 Interleukin-15-Dependent Natural Killer Cell Activation. Mol Cell Biol. 2014;34(6):1158-

$661 \quad 1169$.

662 49. Delconte RB, Kolesnik TB, Dagley LF, et al. CIS is a potent checkpoint in NK cell-

663 mediated tumor immunity. Nat Immunol. 2016;17(7):816-824.

664 50. Holmes ML, Huntington ND, Thong RP, et al. Peripheral natural killer cell maturation 665 depends on the transcription factor Aiolos. EMBO J. 2014;33(22):2721-2734.

666 51. Knox JJ, Cosma GL, Betts MR, Mclane LM. Characterization ofT-bet and Eomes in 667 peripheral human immune cells. Front Immunol. 2014;5:1-13. 
668 52. Shao J, Yin W, Zhang Q, et al. Siglec-7 Defines a Highly Functional Natural Killer Cell

669 Subset and Inhibits Cell-Mediated Activities. Scand J Immunol. 2016;84(3):182-190.

670 53. Jacobs B, Pfefferle A, Clement D, et al. Induction of the BIM Short Splice Variant

671 Sensitizes Proliferating NK Cells to IL-15 Withdrawal. J Immunol. 2019;202(3):736-746.

672 54. Guia S, Jaeger BN, Piatek S, et al. Confinement of activating receptors at the plasma

673 membrane controls natural killer cell tolerance. Sci Signal. 2011;4(167):1-12.

674 55. Islam S, Kjällquist U, Moliner A, et al. Characterization of the single.cell transcriptional

675 landscape by highly multiplex RNA-seq. Genome Res. 2011;21:1160-1167.

676 56. Becht E, McInnes L, Healy J, et al. Dimensionality reduction for visualizing single-cell

677 data using UMAP. Nat Biotechnol. 2019;37(1):38-45.

678 57. van der Maaten L, Hinton G. Visualizing Data using t-SNE. J Mach Learn Res.

$679 \quad 2008 ; 9: 2579-2605$.

680 58. van Dijk D, Sharma R, Nainys J, et al. Recovering Gene Interactions from Single-Cell

681 Data Using Data Difffusion. Cell. 2018;174(3):716-729.

682 59. Levine JH, Simonds EF, Bendall SC, et al. Data-driven phenotypic dissection of AML

683 reveals progenitor- like cells that correlate with prognosis. Cell. 2015;162(1):184-197.

684 60. Kharchenko P V, Silberstein L, Scadden DT. Bayesian approach to single-cell differential 685 expression analysis. Nat Methods. 2014;11(7):18-22.

686 61. Buettner F, Pratanwanich N, McCarthy DJ, Marioni JC, Stegle O. f-scLVM: Scalable and 
Figure 1
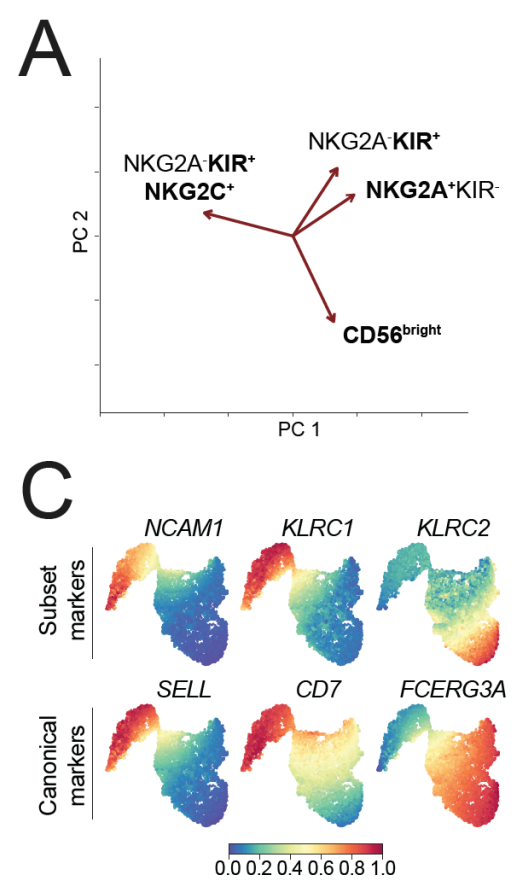

689

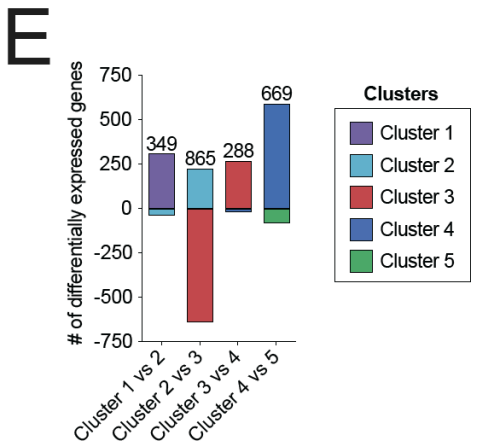

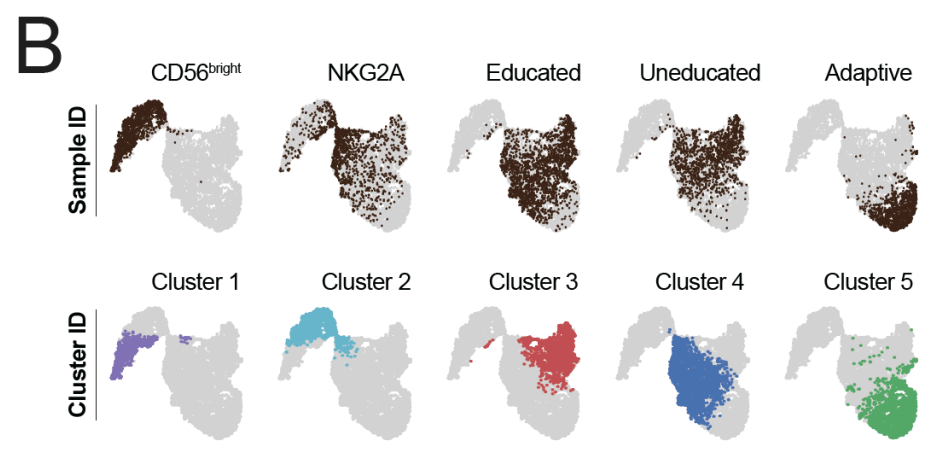
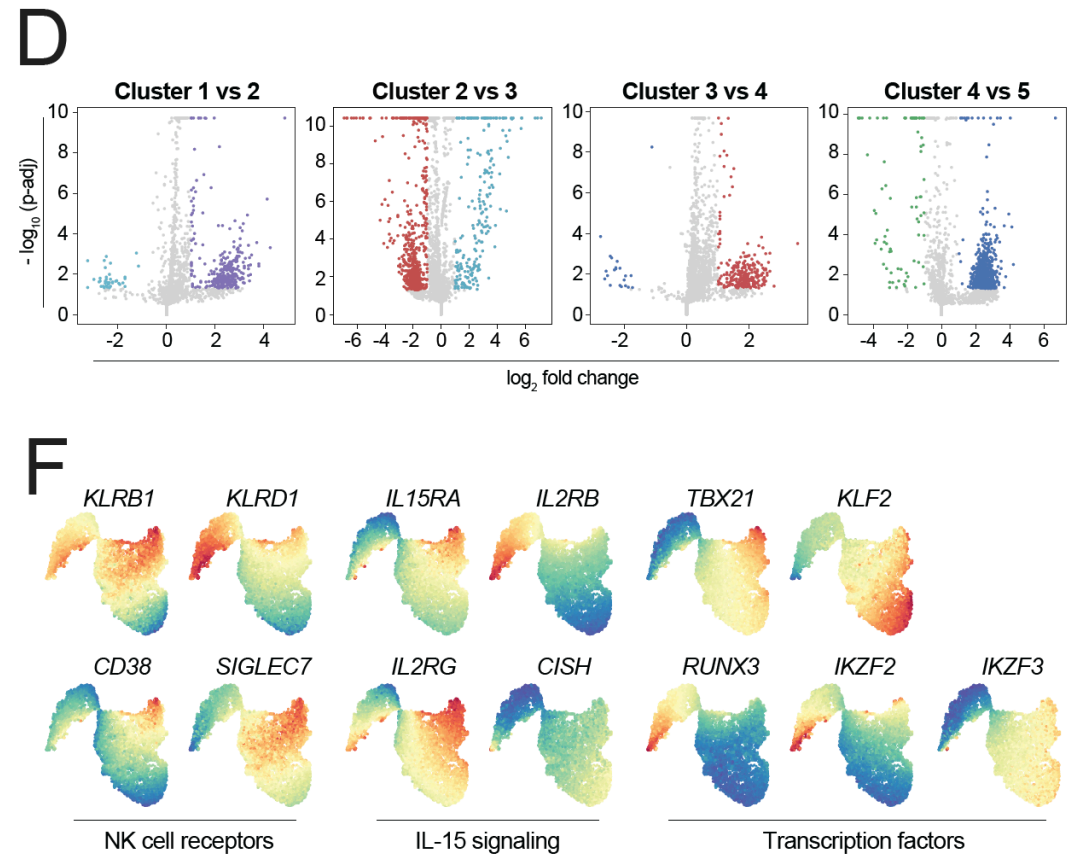

691 Figure 1: NK cell differentiation defined through bulk and single cell RNA-seq

692 (A) PCA plot of bulk RNA seq data of four discrete NK cell differentiation stages. (B) t-SNE plot 693 of scRNA-seq data of five sorted NK cell subsets (CD56 ${ }^{\text {bright }}$, NKG2A ${ }^{+} \mathrm{KIR}^{-} \mathrm{CD}^{-} 7^{-}$CD56 ${ }^{\mathrm{dim}}$, $694 \mathrm{NKG}^{-} \mathrm{A}^{-}$self $\mathrm{KIR}^{+} \mathrm{CD} 57^{-} \mathrm{CD} 56^{\mathrm{dim}}, \mathrm{NKG}^{-} \mathrm{A}^{-}$non-self $\mathrm{KIR}^{+} \mathrm{CD}^{-} 7^{-} \mathrm{CD}^{\mathrm{dim}}, \mathrm{NKG}^{+} \mathrm{A}^{+}$self $\mathrm{KIR}^{+}$ $695 \mathrm{CD}^{+} 7^{+} \mathrm{NKG} 2 \mathrm{C}^{+} \mathrm{CD}^{\mathrm{dim}}$ ) showing transcriptional location of sorted subsets (top row) and 696 PhenoGraph defined transcriptional clusters (bottom row). (C) Gene expression of selected genes 697 displayed as a heatmap on the t-SNE plot. (D) Volcano plots and (E) summary of the number of 
bioRxiv preprint doi: https://doi.org/10.1101/630657; this version posted May 7, 2019. The copyright holder for this preprint (which was not certified by peer review) is the author/funder, who has granted bioRxiv a license to display the preprint in perpetuity. It is made available under aCC-BY-NC 4.0 International license.

698 differentially expressed genes of four comparisons between individual PhenoGraph clusters. (F)

699 Gene expression of selected genes displayed as a heatmap on the t-SNE plot. 


\section{Figure 2}

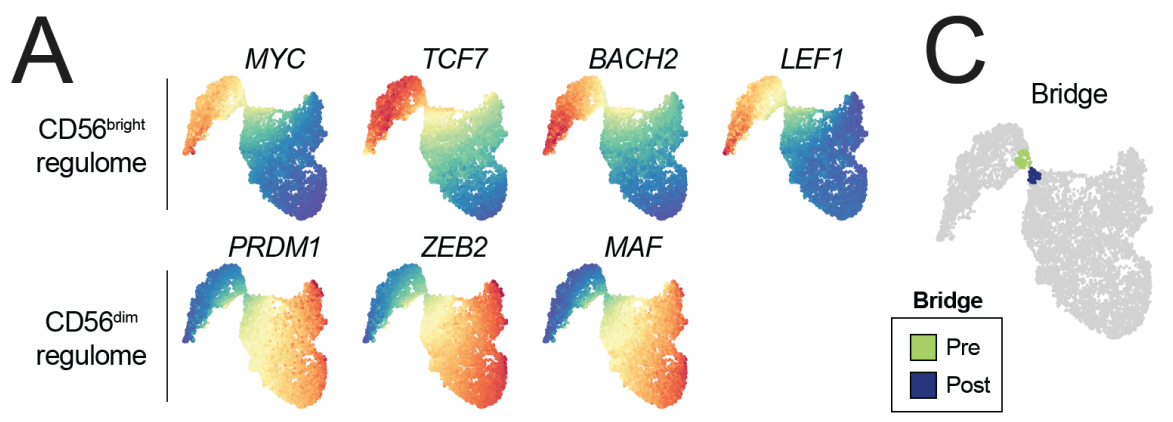

701
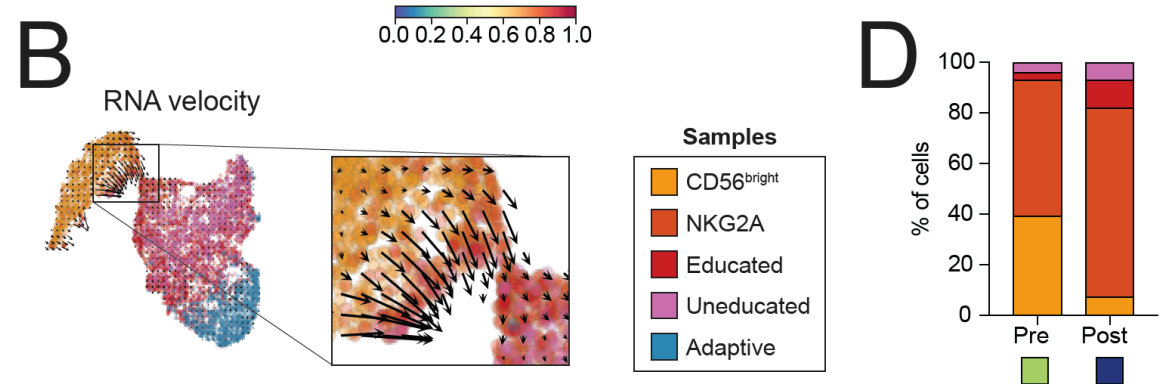

\section{Figure 2: Transitioning from CD56 ${ }^{\text {bright }}$ to CD56 $^{\text {dim }}$ NK cells}

705 (A) Gene expression of selected genes displayed as a heatmap on the t-SNE plot. (B) t-SNE

706 showing sample ID of sorted subsets with the RNA velocity vector overlaid and magnification of

707 the bridge region exhibiting high RNA velocity. (C) Pre (green) and post (blue) bridge region

708 clusters consisting of 100 cells each and defined based on their proximity to the bridge. (D)

709 Frequency of how much each sorted subset contributes to the custom defined bridge clusters. 


\section{Figure 3}

\section{A}

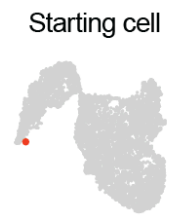

Pseudotime

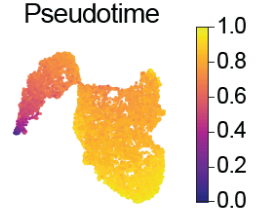

Terminal cells

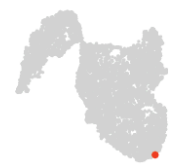

\begin{tabular}{|c|}
\hline \multicolumn{1}{|c|}{ Clusters } \\
\hline$\square$ Cluster 1 \\
\hline$\square$ Cluster 2 \\
$\square$ Cluster 3 \\
$\square$ Cluster 4 \\
$\square$ Cluster 5 \\
\hline
\end{tabular}

B

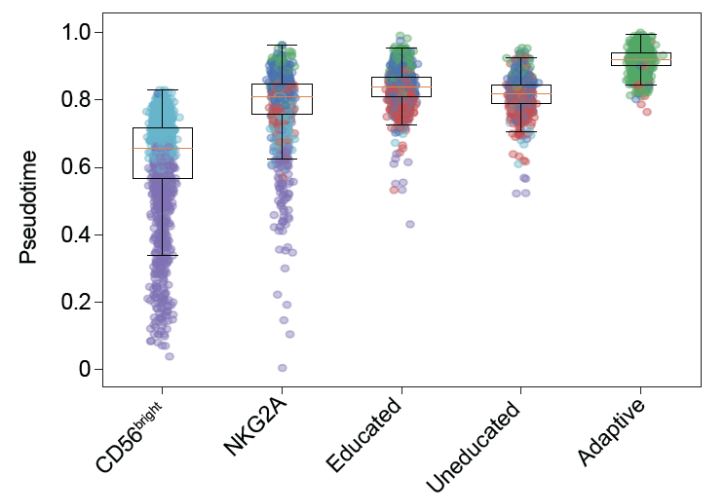

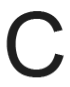

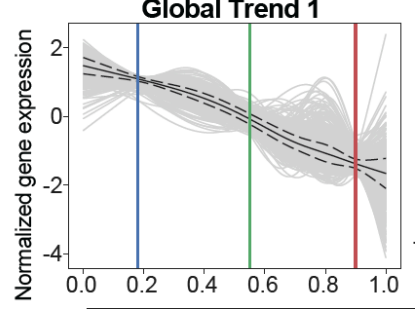

Global Trend 1
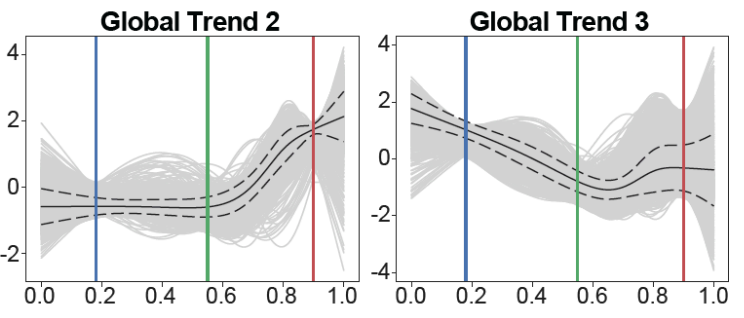

$\mathrm{D}$

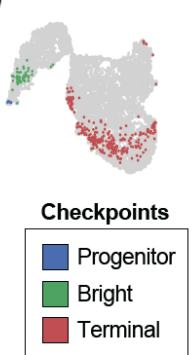

Pseudotime

E

mitochondrial translational elongation

neutrophil degranulation regulation of hematopoietic stem cell differentiation

TNF-mediated signaling pathway stimulatory C-type lectin receptor signaling pathway
Global Trend 1

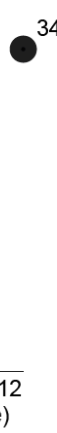

Global Trend 2

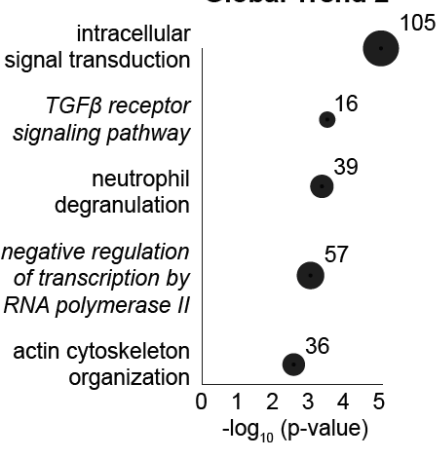

Global Trend 3

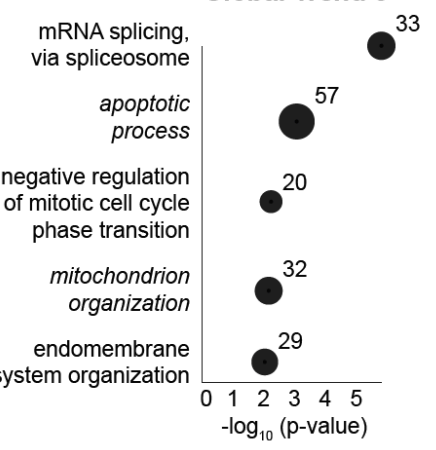




\section{Figure 3: Differentiation checkpoints at distinct stages of pseudotime}

713 (A) Starting cell (highest MYC expression), terminal cell and pseudotime as calculated using the

714 Palantir algorithm. (B) Distribution of each sorted subset in pseudotime with colors denoting the

715 PhenoGraph clusters each cell identifies with (box limits, upper and lower quartiles; center line,

716 median; whiskers, quartile $1-1.5 *$ inter quartile range to quartile $3+1.5 *$ inter quartile range;

717 points, outliers). (C) Global gene trends mapped onto pseudotime with colored lines denoting

718 transcriptional checkpoints defined by low standard deviation (blue $=$ progenitor, green $=$ bright,

719 red = terminal). (D) Visualization of cells corresponding to each checkpoint identified in

720 pseudotime. (E) Gene set enrichment analysis of selected significant gene ontology (GO) terms

721 associated with each gene trend. Significance was calculated using Fisher's exact test followed by

722 false discovery rate correction of the $p$-value. A negative $\log _{10}$ value of the false discovery rate

723 adjusted $p$-value $>1.3$ is deemed significant. 


\section{Figure 4}

725
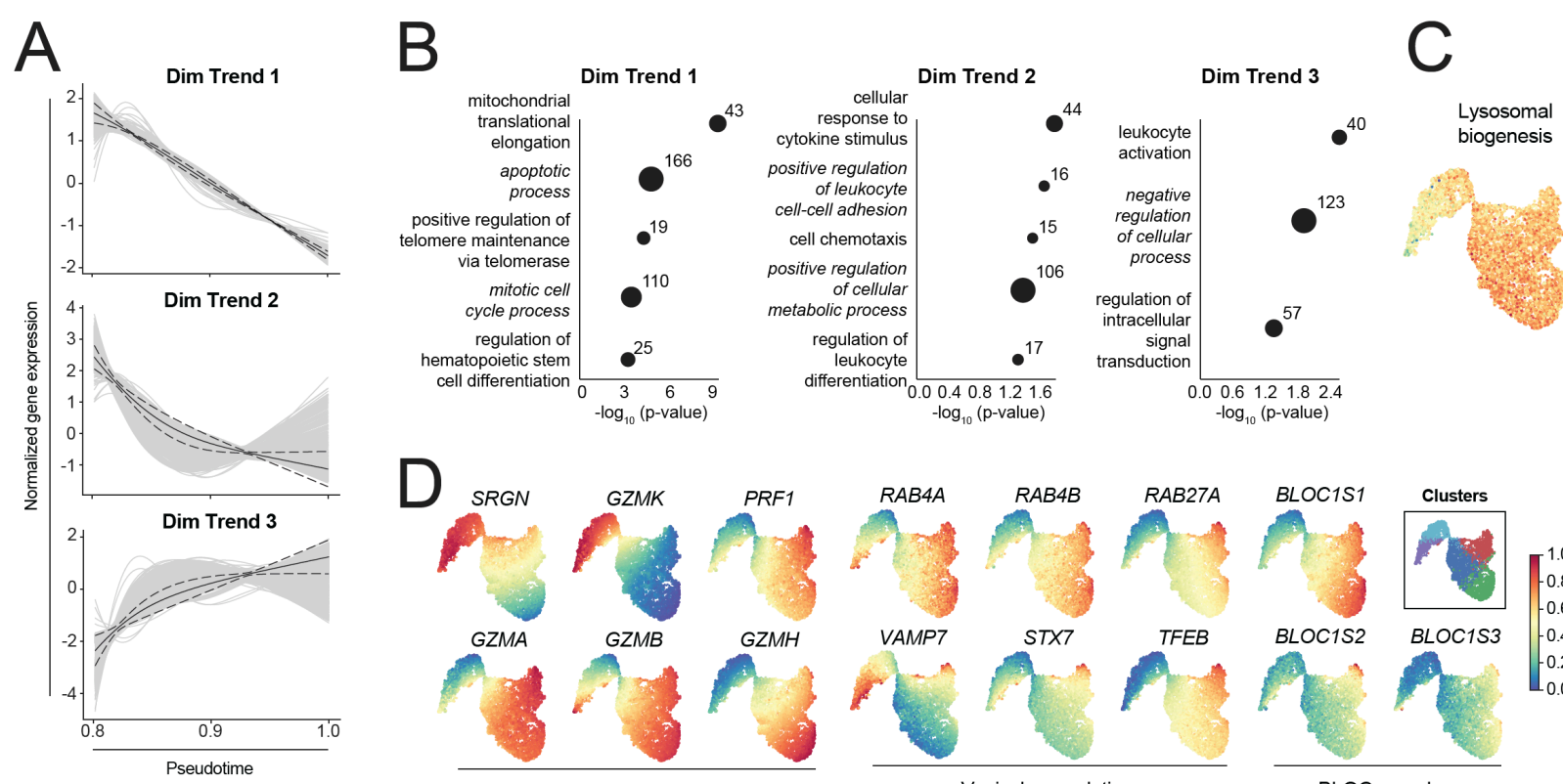

Vesicular components

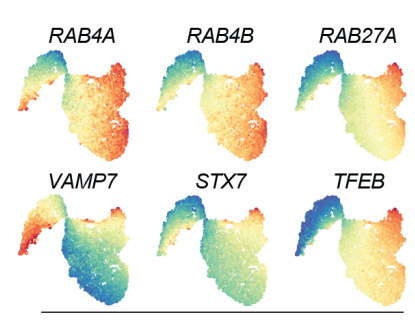

Vesicular regulation

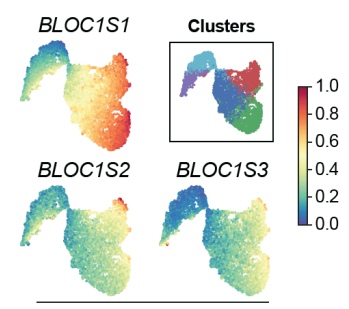

BLOC complex

Figure 4: Establishment of a functional template during CD56 $^{\mathrm{dim}}$ NK cell differentiation

729 (A) Dim gene trends mapped onto pseudotime, only taking pseudotime $>80 \%$ into account.

730 (B) Gene set enrichment analysis of selected significant gene ontology (GO) terms associated with

731 each gene trend. (C) Factor analysis of genes associated with lysosomal biogenesis. (D) Gene

732 expression of selected genes displayed as a heatmap on the t-SNE plot. Significance was calculated

733 using Fisher's exact test followed by false discovery rate correction of the p-value. A negative $\log _{10}$

734 value of the false discovery rate adjusted $p$-value $>1.3$ is deemed significant. Numbers in the plot

735 represent number of genes identified within each GO term based on the DEGs analyzed. 